\title{
Vorhofflimmern
}

\section{Antiarrhythmikum reduziert Schlaganfallrisiko}

— Die Therapie des Vorhofflimmerns (VF) mit Dronedaron reduziert das relative Risiko für Schlaganfälle um ein Drittel, wie eine Post-hoc-Analyse der ATHENA-Studie zeigt.

Vorhofflimmern ist die häufigste Arrhythmie des Herzens und einer der wichtigsten Risikofaktoren für den Schlaganfall, die bei diesen Patienten oft besonders schwerwiegend sind. Auch das Demenzrisiko ist erhöht: „Wir gehen davon aus, dass Vorhofflimmern - wahrscheinlich über leichte, unbemerkte Hirninfarkte - langfristig die kognitiven Funktionen der Patienten beeinträchtigt", so Priv.-Doz. Joachim Ehrlich, Frankfurt.

Ein essenzieller Aspekt der Therapie von Vorhofflimmern ist daher die Prävention kardioembolischer Ereignisse. In Ab- hängigkeit vom individuellen Risiko, das über den $\mathrm{CHADS}_{2}$-Score oder heute besser mit dem $\mathrm{CHADS}_{2}$-VASC-Score bestimmt wird, muss der Patient dazu antikoaguliert werden.

Auch die Therapie des Vorhofflimmerns mit Dronedaron, basierend auf leitliniengerechter Antikoagulation, kann zur Vermeidung von Schlaganfällen beitragen. Wie Ehrlich berichtete, verringerte Dronedaron in der ATHENA-Studie das Schlaganfallrisiko um 34\%, wobei Patienten mit einem $\mathrm{CHADS}_{2}$-Score $>2$ besonders profitierten.

\section{ESC-Leitlinien-Empfehlung}

Dronedaron ist als Multaq ${ }^{\circledR}$ in der EU bei erwachsenen, klinisch stabilen Patienten mit nicht permanentem Vorhofflimmern zugelassen, um ein Wiederauftreten von VF zu verhindern oder die ventrikuläre Herzfrequenz zu senken. Die Leitlinien der European Society of Cardiology empfehlen das Antiarrhythmikum als Therapieoption der ersten Wahl für den Erhalt des Sinusrhythmus bei Patienten mit nicht permanentem Vorhofflimmern - ausgenommen Patienten mit chronischer Herzinsuffizienz in NYHA-Klassen III und IV sowie instabiler Herzinsuffizienz mit Dekompensation innerhalb des letzten Monats.

- Jürgen Setton

Quelle: Satellitensymposium „Teaching Course

- Rhythmologie für Neurologen“, 28. Jahres-

tagung von DGNI und DSG (ANIM), Leipzig,

Januar 2011 (Veranstalter: Sanofi-Aventis)

\section{Depressive Störungen im Alter}

\section{Serotonin-Wiederaufnahmehemmer bieten Vorteile}

\begin{abstract}
— "Liegt die durchschnittliche Häufigkeit depressiver Erkrankungen in der Bevölkerung bei $5 \%$, so müssen wir mit steigendem Lebensalter von deutlich höheren Zahlen ausgehen", betonte Dr. Michael Enzl, Münster. Besonders gefährdet sind Patienten mit körperlichen Gebrechen und Immobilität.
\end{abstract}

\footnotetext{
Ältere Menschen haben oft Angst vor einer psychiatrischen Diagnose

Doch im Alter wird die Depression häufig nicht erkannt. Gerade ältere Menschen haben Angst vor einer psychiatrischen Diagnose. Dazu kommt, dass sich die Symptomatik der Altersdepression wesentlich von der bei Jüngeren unterscheidet. „Der ältere Patient klagt eher über wechselnde körperliche Beschwerden und verneint auch auf Nachfragen Traurigkeit und Resignation", so Enzl. Eine besondere Schwierigkeit ist die Abgrenzung depressiver Störungen von einer beginnenden Demenz, oder sie bei Demenz zu erkennen.

Die Depression im Alter darf keinesfalls unbehandelt bleiben, da depressive Störun-
}

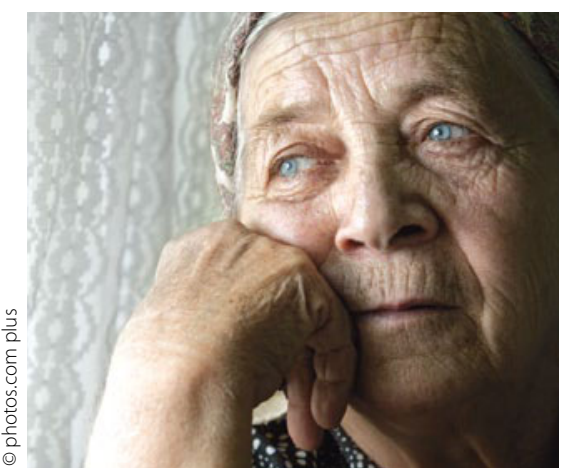

Bei der Wahl des Antidepressivums auf nicht sedierende Wirkung achten.

gen auch einen erheblichen Einfluss auf den Genesungsprozess von körperlichen Erkrankungen haben, $z$. B. auf den Rehabilitationsprozess nach Schlaganfall oder Herzinfarkt.

Bei der Wahl des Antidepressivums müssen verschiedene Gesichtspunkte berücksichtigt werden. Das Medikament sollte nach Möglichkeit keine sedierende Nebenwirkung entfalten, um das Risiko von Stürzen nicht zu erhöhen. Auch anticholinerge Nebenwirkungen mit Sehstörungen, Obstipation und Verschlechterung der kognitiven Funktionen, wie sie bei trizyklischen Antidepressiva beobachtet werden, sind für den Patienten sehr belastend.

"Als besonders gut wirksam und verträglich haben sich gerade bei der Altersdepression moderne Antidepressiva, nämlich die Serotonin-Wiederaufnahmehemmer bewährt", so Enzl. Dabei handelt es sich nicht um eine homogene Substanzgruppe. Vielmehr bestehen erhebliche Unterschiede hinsichtlich Verträglichkeit und Interaktionen zwischen den verschiedenen Substanzen. Für Escitalopram (Cipralex ${ }^{\circledast}$ ) spreche seine gute Wirksamkeit bei guter Verträglichkeit.

Da insbesondere ältere Patienten ein erhöhtes Rezidivrisiko zeigten, ist häufig eine längerfristige Einnahme des Antidepressivums über Jahre erforderlich, sodass auch im Hinblick auf die Compliance ein geringes Nebenwirkungsspektrum für den Therapieerfolg entscheidend ist.

\footnotetext{
- Dr. med. Peter Stiefelhagen

Quelle: Satellitensymposium im Rahmen des DGIM-Kongresses, Wiesbaden, April 2011 (Veranstalter: Lundbeck)
} 